\title{
La dimensión educativa y solidaria del voluntariado en la Universidad Nacional del Litoral
}

\section{Marcel Mónica Blesio}

Directora del Sistema Integrado de

Programas y Proyectos de Extensión

de la Secretaría de Extensión,

Universidad Nacional del Litoral,

Argentina.

mblescio@unl.edu.ar

\author{
Marcelo Mendoza \\ Coordinador del Programa del \\ Voluntariado de la Secretaría de \\ Extensión, Universidad Nacional \\ del Litoral. \\ mmendoza@fcjs.unl.edu.ar
}

Integración de la docencia y la extensión /

Desafíos de gestión

RECEPCIÓN: 24/06/16

ACEPTACIÓN FINAL: 16/10/16

\section{Resumen}

La intención de promover la participación de toda la comunidad universitaria fundamentalmente de estudiantes de nuestra universidad en dispositivos institucionales existentes, plantea desafíos importantes para la gestión académica. La decisión de ampliar y enriquecer el concepto de prácticas voluntarias y solidarias, incorporando la dimensión educativa y pedagógica que ellas encierran, ha marcado diferencias con concepciones más tradicionales del voluntariado que también tienen vigencia.

Este artículo se propone recuperar la experiencia de más de una década del Programa del Voluntariado en la Universidad Nacional del Litoral, así como hacer explícitos los fundamentos de su creación, el andamiaje normativo, las perspectivas teóricas donde se sustenta y los dispositivos de gestión desarrollados para llevar adelante esta política universitaria.

\section{Resumo}

A intenção de promover a participação de toda a comunidade universitária, principalmente dos estudantes da nossa universidade em dispositivos institucionais existentes, apresenta desafios importantes à gestión acadêmica. A decisão de ampliar e enriquecer conceito de práticas voluntárias e solidárias, incorporando a dimensão educativa e pedagógica que elas abrangem, marcou diferenças com concepções tradicionais do voluntariado que também estão em vigência.

Este artigo propõe recuperar a experiência de mais de uma década do Programa do Voluntariado na Universidad Nacional del Litoral, assim como explicitar os fundamentos de sua origem, o suporte normativo, as perspectivas teóricas em que se sustenta e os dispositivos de gestão desenvolvidos para levar adiante esta política universitária.

Palavras-chave

- Voluntariado

- Institucionalização

- Educação experiencial

- Solidariedade
Para citación de este artículo Blesio, M. y Mendoza, M. (2016). La dimensión educativa y solidaria del voluntariado en la Universidad Nacional del Litoral. En Revista +E versión digital, (6), pp. 164-171. Santa Fe, Argentina: Ediciones UNL. 


\section{A modo de introducción}

El recorrido realizado desde la creación del Programa del Voluntariado Universitario permite poner en evidencia la contribución importante realizada para el fortalecimiento del entramado de las tres funciones sustanciales de la universidad: docencia, investigación y extensión.

La creación de este Programa tiene como antecedente el trágico suceso de las inundaciones en el año 2003 en la ciudad de Santa Fe, cuando el río Salado avanzó sobre el entramado urbano, cubrió viviendas, espacios verdes, centros recreativos y hasta hospitales, lo que alteró de manera dramática la vida cotidiana de miles de santafesinos. Ante esta situación, la población no afectada en general y la comunidad universitaria en particular reaccionaron de manera solidaria ofreciendo su colaboración. Para ello fue necesaria la planificación de acciones por parte de la UNL y la Federación Universitaria del Litoral (FUL). Desde donde se organizaron tales acciones vinculadas a ordenar tanto la participación de estudiantes como del resto de la comunidad universitaria.

La experiencia vivida en esos meses no quedó en el anecdotario sino que sirvió para demostrar la capacidad de organización de una fuerza voluntaria que reunió, sin diferencias, a autoridades, personal docente, no docente, graduados, estudiantes y ciudadanos. Fue el puntapié inicial para que se sustancie la Resolución del CS $n^{\circ} 76$ del año 2004 que reconoce la figura del voluntario y crea el Programa del Voluntariado Universitario. El Programa plantea como objetivo central la promoción de la participación responsable y solidaria de la comunidad académica en articulación con la sociedad civil en pos de abordar necesidades y problemáticas sociales. En este marco, la Universidad interviene a través de proyectos y programas de extensión diseñados para actuar en el marco de un proceso dialógico de generación y apropiación de conocimientos. A partir de ese momento, la extensión y el voluntariado comienzan a manifestarse en la vida de la comunidad universitaria, fundamentalmente en el estudiantado iniciando una etapa de participación institucionalizada en Proyectos y Programas de Extensión, en consonancia con los objetivos planteados por la mencionada normativa.

Dando cuenta de esta convicción, en el año 2007, un grupo de consejeros estudiantiles, movilizados por el interés de instaurar condiciones institucionales que favorezcan la incorporación de las prácticas de extensión a las propuestas curriculares de las carreras de la UNL, presentó un proyecto que se materializó en la Resolución CS $n^{\circ} 274$, que plantea como objetivo profundizar alternativas de formación de profesionales sólidos y fundamentalmente comprometidos con la sociedad. Entre sus principales expresiones resaltamos:

"La participación de alumnos en las prácticas de extensión los pone en contacto con una realidad que los coloca en situación de asumir una visión solidaria del conocimiento que fueron construyendo en la Universidad y que les implica abordajes de tipo profesional. Estas experiencias significan la asunción de una perspectiva preocupada por la formación de profesionales críticos, conscientes de su compromiso social en el ejercicio profesional y requiere de una visión pedagógica que aborde la práctica y favorezca procesos de reflexión sobre las vivencias reconstruidas sistemática y conceptualmente, con el objeto de someterlas a niveles crecientes de teorización".

Sin dudas, estas vivencias aportan a la formación integral de sujetos responsables, con conciencia ética y solidaria, crítica y reflexiva, capaces de mejorar la calidad de vida de la comunidad.

\section{Reflexiones sobre el valor pedagógico, educativo y solidario del voluntariado}

El aula no parece un lugar apropiado para considerar los sentimientos, ni las experiencias personales, ni los intercambios intersubjetivos de quienes trabajan en ella. Se olvida, como dice Morin, que "existe un conocimiento que es comprehensivo y que se funda sobre la comunicación, la empatía, incluso la simpatía intersubjetiva" (2000:122-123).

Nos interesa reflexionar sobre esta concepción, que consideramos está estrechamente vinculada con las prácticas de educación experiencial. Hoy, en diversos ámbitos universitarios, hablamos de la importancia de la incorporación de este tipo de educación como una estrategia de aprendizaje que aporta tanto a la formación del estudiantado como a la docencia. Es oportuno, entonces, preguntarnos y reflexionar sobre el valor pedagógico de la inclusión de la educación experiencial en la universidad. En este sentido, Camilloni sostiene:

"La educación experiencial es una estrategia de enseñanza con enfoque holístico, que está destinada a relacionar el aprendizaje académico con la vida real. Con ese fin, propone al alumno realizar actividades en las que a partir de esa conexión con la práctica, ponga a prueba, en situación auténtica, es decir, de la vida real, las habilidades y conocimientos teóricos que posee, evalúe sus consecuencias, enriquezca esos conocimientos y habilidades identifique nuevos problemas. Cuando el aprendizaje es experiencial, quien aprende lo hace en las condiciones sociales de la utilización auténtica de los conocimientos. (...) Para que un programa de educación experiencial, en sus variadas formas pueda configurarse de manera adecuada, la universidad, debe crear condiciones que permita una rápida toma de decisiones en cuanto a la apertura y cierre de actividades, es preciso que establezca redes de relaciones con instituciones 'socias', que faciliten la rápida adaptación de estudiantes y tutores. David Kolb es un referente fundamental para comprender los alcances del concepto de educación experiencial. Entre las diversas modalidades de la educación experiencial, se encuentra el aprendizaje-servicio 


\section{6}

conceptualizar al voluntariado

desde la perspectiva de la

extensión universitaria implica

ir más allá de la simple voluntad

manifestada en actividades

nobles y solidarias

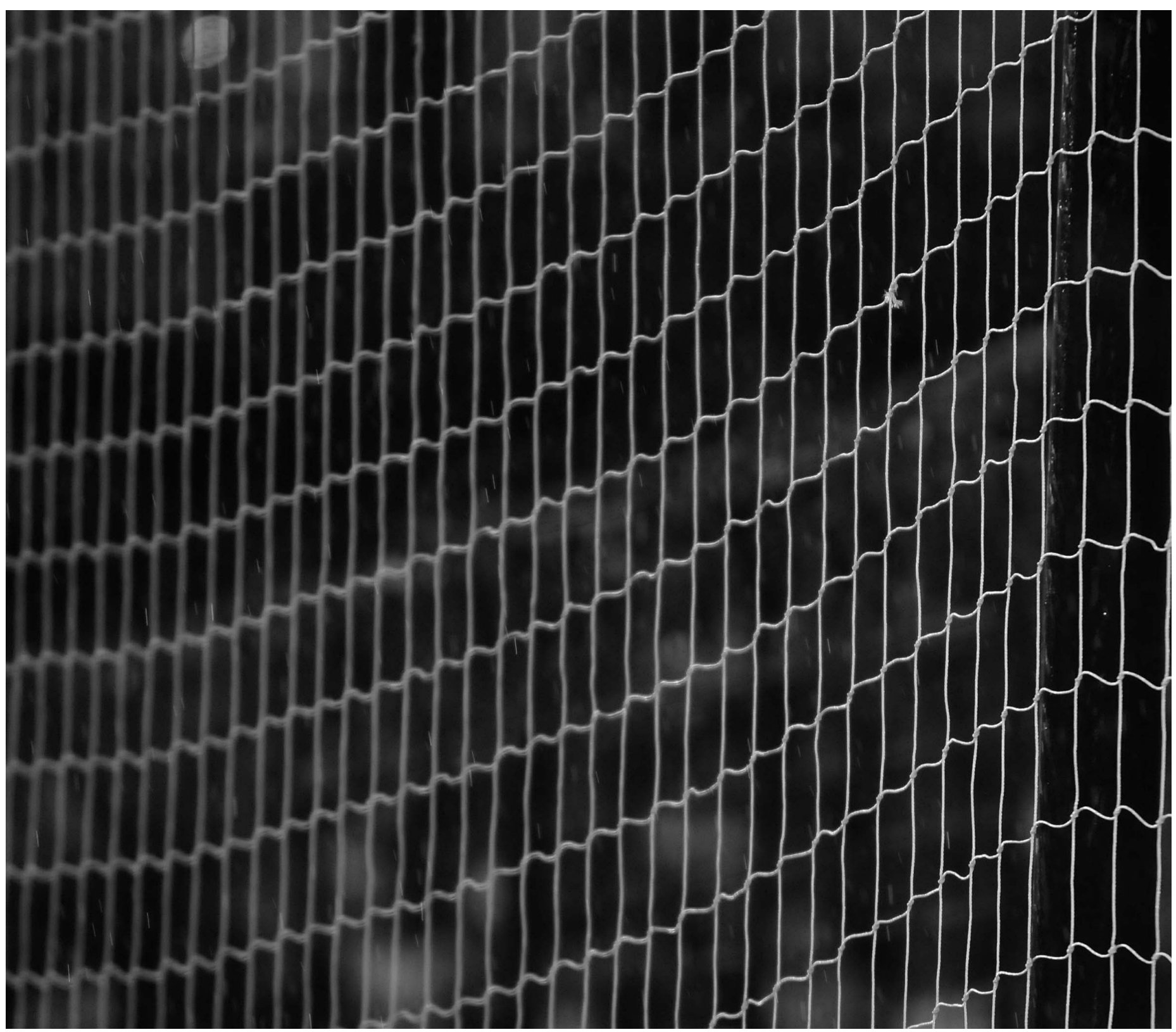

(c) Hugo Pascucci 
que es una estrategia de enseñanza en la que los estudiantes aplican sus habilidades y conocimientos académicos y profesionales específicos para satisfacer necesidades reales en respuesta a requerimientos explícitos de la comunidad. Además de servir a la formación profesional, el aprendizajeservicio contribuye a la educación para la ciudadanía y la formación ética de los estudiantes. (...) el aprendizajeservicio pone el acento en los dos términos que constituyen esta expresión, el aprendizaje y el servicio, y no sólo en uno de ellos como sería la práctica profesional que se centra en el aprendizaje". (2001:78)

Una manera de graficar los alcances del aprendizaje-servicios y diferenciarlos de otras acciones educativas desarrolladas en un contexto comunitario, son los "cuadrantes" (ver Cuadro 1). Estos "cuadrantes del aprendizaje-servicio" fueron desarrollados originalmente por especialistas de la Universidad de Stanford. El esquema tradicional de los cuadrantes comenzó aplicándose a instituciones educativas, y es el siguiente:

\section{Cuadro 1}

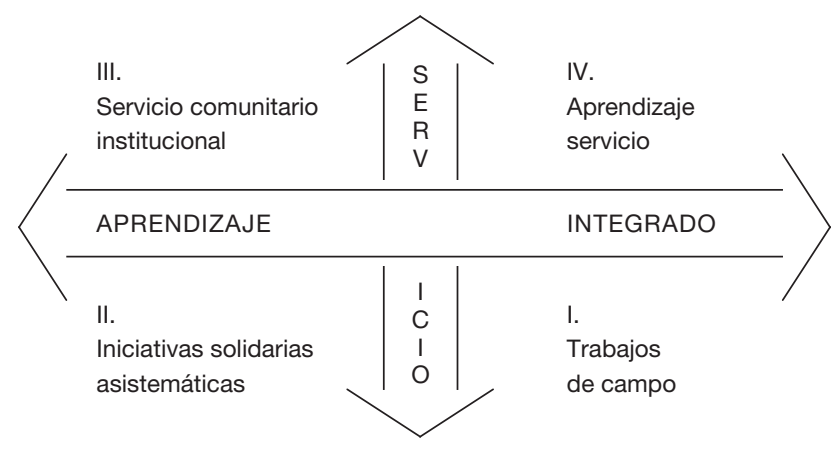

Podemos observar que el eje vertical nos habla de la menor o mayor calidad del servicio solidario que se presta a la comunidad y el eje horizontal indica la menor o mayor integración del aprendizaje sistemático o disciplinar al servicio que se desarrolla. En función de estos cuadrantes, en la mitad inferior ubicamos a los proyectos con menor calidad de servicio o de aprendizaje sistemático. En nuestro caso, en estos últimos años se ha venido instalando la necesidad de valorizar y enriquecer estas prácticas solidarias promovidas desde el programa de voluntariado, en las que participan cientos de estudiantes de diversas carreras, donde no solo se pone en juego el "servicio" sino la dimensión pedagógica y educativa de esta participación. Los programas y proyectos de extensión se constituyen en espacios privilegiados que ofrecen diversos campos de conocimiento para que estudiantes y otros miembros de la comunidad universitaria realicen prácticas voluntarias, constituyéndose en opciones éticas, humanísticas, que sin dudas tendrán efectos en la formación profesional. El encuentro fuera del aula entre profesores/as y alumnos/as, en espacios abiertos, en escenarios reales a partir de la experiencia vivida, da lugar al encuentro con lo personal y lo intersubjetivo. Aquí, la confianza y el respeto se hacen presentes y favorecen el desarrollo de actitudes y valores en la formación de ciudadanos y ciudadanas responsables, capaces de contribuir al mejoramiento de la calidad vida de la comunidad. La incorporación de voluntarios no solo apela a una conciencia ética y solidaria sino también a que haya reflexiones críticas sobre las problemáticas sociales.

\section{Institucionalización e implementación del voluntariado como una práctica educativa y solidaria en la UNL}

Habiéndose explicitado los fundamentos del punto anterior, es posible indicar que en los últimos años, en varias oportunidades la denominación de "voluntariado" ha generado discusiones sobre la pertinencia del término. Si acudimos a la Real Academia Española se define como el "conjunto de personas que se ofrecen voluntariamente a realizar algo". El término "voluntariado" es el más utilizado internacionalmente a la hora de referirse a personas que realizan actividades en forma voluntaria, no obligatoria, donde se supone que no existen demasiadas regulaciones ni exigencias, justamente por este carácter de "voluntario". Podríamos interpretar que esta condición de ser voluntario corre el riesgo de agotarse, extinguirse, y nos hizo repensar ese concepto que es universalmente utilizado para identificar personas que realizan tareas solidarias, sacrificadas, intencionales, sin exigencias de recompensas económicas, para agregarle nuevos valores. De allí que este concepto fue enriqueciéndose con matices que hacen a la jerarquización del voluntario/a como tal, otorgándole una impronta reformista desde una faz subjetiva. En tal sentido, conceptualizar al voluntariado desde la perspectiva de la extensión universitaria implica ir más allá de la simple voluntad manifestada en actividades nobles y solidarias. El reformismo supone que la Universidad debe promover su función social desarrollando la extensión. No es voluntarismo el que moviliza a la formación, transferencia y transformación del conocimiento científico en un proceso de interacción con el medio que la circunda, sino la convicción, la voluntad entendida como el carácter, el arrojo, el compromiso que identifican el "deber ser" de la Universidad Reformista. Para que ello ocurra se requieren políticas institucionales que apunten a la realización de prácticas y experiencias educativas, que se asienten en principios que apuesten al desarrollo integral de los profesionales. Así lo demuestra la UNL al reafirmar este principio en los documentos institucionales y en la Resolución del CS n 76/04, que promueve la participación responsable y solidaria de la comunidad universitaria en articulación con los distintos espacios 
de la sociedad civil en el desarrollo de los Proyectos de Extensión y las Acciones de Extensión al Territorio. Esta participación se sostiene en la convicción de que el buen profesional no es aquel que sólo domina su campo de conocimiento, sino precisamente el que además tiene una actitud solidaria ante lo social, desde una mirada ética que reconozca otras realidades que afectan a grandes franjas de la población. Se trata de brindar espacios donde pueda ponerse a prueba en situación auténtica, las habilidades y conocimientos teóricos aprendidos en las aulas.

Estos conceptos han sido reafirmados últimamente en documentos institucionales aprobados, como: el nuevo estatuto -2012- y el Plan de Desarrollo institucional -2010-2019—, donde se observa una clara preponderancia de lo social en la visión de la Universidad sobre qué es el conocimiento, el aprendizaje y la enseñanza. Se intenta así problematizar la idea del conocimiento vinculado únicamente a lo intelectual y se enfatiza el conocimiento como un proceso en el que intervienen la inteligencia, el deseo, el cuerpo, los modos en que se interactúa con otros, donde lo afectivo, lo emocional, la empatía encuentran un lugar fundamental en la acción y recuperan las teorías de Dewey, que establecían esta conexión orgánica entre educación y experiencia personal. Camilloni nos plantea que cuando el aspecto cognitivo se integra al afectivo y da lugar a la acción, entonces se puede hablar de "aprendizaje experiencial".

En este orden de ideas, la sanción del Código de Ética aprobado por Resolución CS n 168 del año 2004, junto a la resolución de creación del Programa Voluntariado Universitario, abrió la posibilidad de que tanto docentes como estudiantes, graduados y no docentes participen de modo programático y orgánico en los distintos proyectos de extensión y otras acciones en materia de extensión social. En el Programa surge un plexo normativo que contempla "deberes del voluntariado hacia los beneficiarios", "deberes del voluntario hacia la/s organización/es convocantes", "deberes del voluntario hacia los otros voluntarios", "deberes del voluntario hacia la sociedad" y "derechos de los voluntarios". En la actualidad, el voluntariado universitario forma parte del Sistema Integrado de Programas y Proyectos de Extensión ${ }^{1}$ como dispositivo de gestión transversal para la realización de acciones de extensión regulares y sistemáticas que se vehiculizan en el centronorte de la provincia de Santa Fe. Desde su implementación a la fecha se han ido diseñando diferentes instancias que responden a necesidades formales, administrativas y pedagógicas, que permiten fortalecer la institucionalización de esta práctica voluntaria, acentuando su carácter solidario y su potencial educativo. Desde la coordinación del Programa se diseñaron diversos dispositivos de gestión que se traducen en actos administrativos para dar marco formal a la propuesta de participación. Incorporarse como voluntario en un programa o proyectos de extensión implica para todos los involucrados asumir un compromiso, que se traduce en cumplir exigencias, requisitos y pautas establecidas con anticipación y que son condición indispensable para el reconocimiento y certificación final, como son: fichas de inscripción, acta acuerdo, informes de evaluación y autoevaluación, entre otras. Cada uno de estos instrumentos fue diseñado en pos de recuperar las experiencias con la mayor cantidad y calidad de información.

La participación del estudiantado requiere por parte de los docentes de una minuciosa planificación con tareas pensadas para el logro de los objetivos planteados, que permita a quienes se desempeñan como voluntarios/as múltiples experiencias de aprendizaje así como aproximaciones y valoraciones sobre su desempeño en el proyecto o programa de referencia. En cuanto a la evaluación de la experiencia, desde la coordinación del Programa se solicita la confección de informes por parte de los voluntarios planteados de acuerdo con la duración de la propuesta y que tienen por objetivo registrar las vivencias y aprendizajes en sus diferentes dimensiones: técnicas, académicas, personales. Para lo cual se hizo necesario desarrollar guías que acompañen el proceso vivido en busca de sistematizar información, tanto en aspectos cognitivos como personales, vinculadas a aspectos emocionales y afectivos. Asimismo, la dirección del Proyecto o Programa de extensión incorpora, durante el desarrollo y hasta finalizar el proyecto, estrategias de evaluación. Las perspectivas que consideramos más adecuadas para evaluar las prácticas del voluntariado son aquellas que proveerán al estudiante de elementos para mejorar su desempeño valorando su participación, su contribución al proyecto, su integración al equipo, las que indiquen las actividades desarrolladas por los mismos así como el grado de compromiso manifestado. Nos parece válido recuperar la perspectiva formativa de la evaluación, y en este sentido Álvarez Méndez sostiene:

"la evaluación formativa no inspira miedo o sentimiento del deber cumplido vinculado a la idea de acreditación sino que propicia que los alumnos desarrollen la inquietud y la curiosidad por seguir aprendiendo, que la evaluación se convierta en una "vía de emancipación y de investigación". (2003:155)
1) Sistema Integrado de Programas $y$ Proyectos, Res. CS n 192/06: (SIPPE): se constituye como un instrumento de gestión que contiene propuestas de innovación con intervención social y forma parte de la Política de Extensión Universitaria de la UNL. 


\section{4}

Incorporarse como voluntario

implica asumir un compromiso,

que se traduce en cumplir

exigencias, requisitos y pautas

establecidas con anticipación

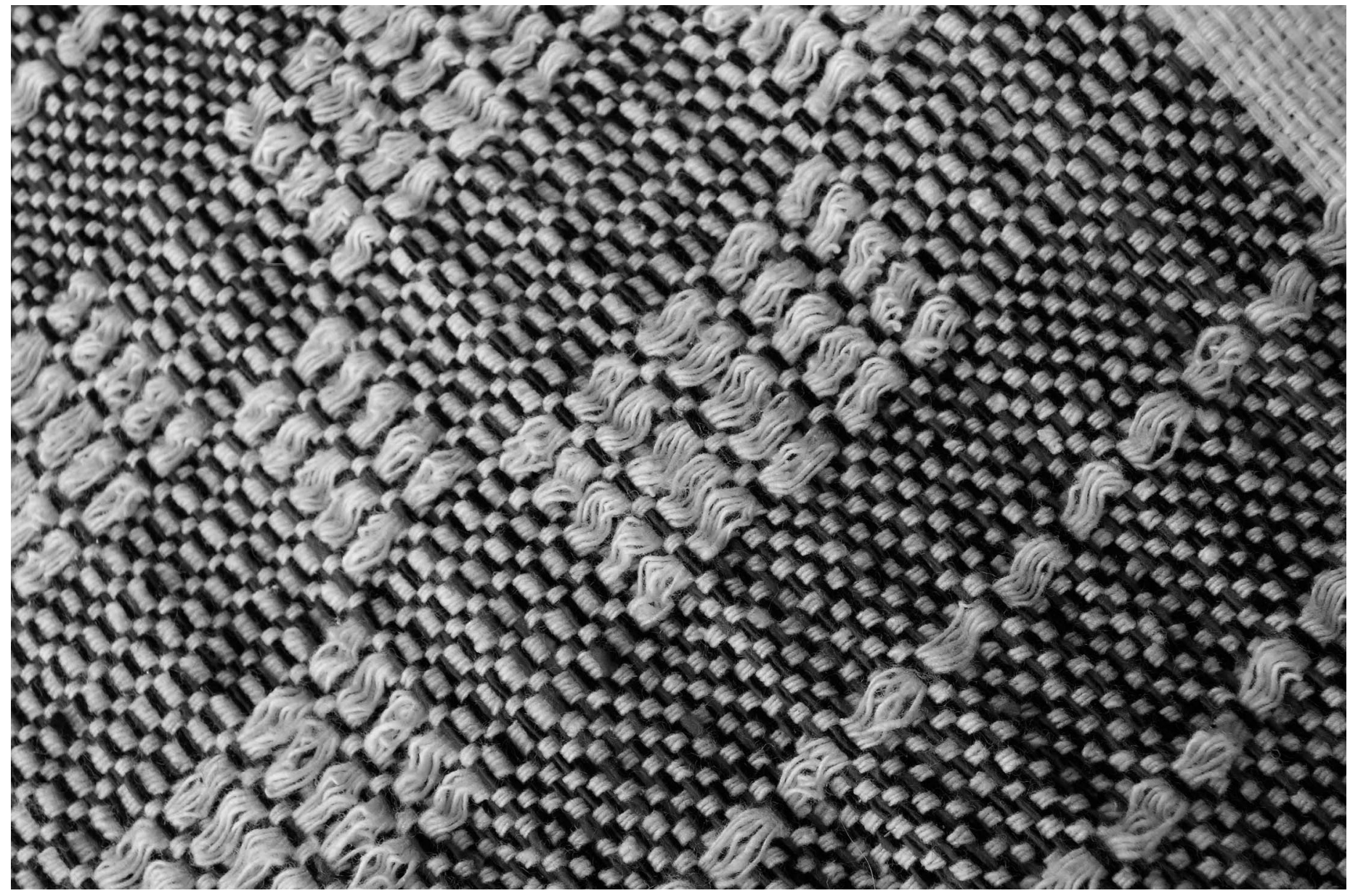

(C) Micaela Block 
Asimismo, Rafaghelli nos aporta:

"las prácticas extensionistas, como prácticas sociales, interculturales, son propicias para las evaluaciones comprehensivas, hermenéuticas e interpretativas, donde la preocupación no está en medir resultados o datos, sino que se preocupan por realizar una interpretación situacional del sentido que los actores otorgan al proyecto en el cual participan". (2013:33)

Los desafíos que afrontamos son enormes: cientos de estudiantes participan en los diferentes dispositivos diseñados por la Secretaría de Extensión. Todas estas propuestas se presentan como verdaderas situaciones auténticas donde se ponen a prueba las habilidades y conocimientos teóricos aprendidos en las aulas pero donde también se manifiestan otros valores vinculados a la solidaridad, la empatía, el compromiso social, la afectividad. Pero hay un elemento central en estas prácticas que se enmarcan en el voluntariado, y se trata del conocimiento académico, que es lo que las distingue de cualquier otra práctica. Decimos que son prácticas solidarias porque tienen lugar en un contexto de compromiso social, enmarcadas en una formación integral que favorece el desarrollo de actitudes y valores en la formación de ciudadanos y ciudadanas responsables, con conciencia ética y solidaria, crítica y reflexiva, capaces de mejorar la calidad de vida de la comunidad. A continuación presentamos dos gráficos confeccionados con datos disponibles en la Dirección del Sistema Integrado de Programas y Proyectos (SIPPE) y elaborados por el área de Coordinación del PVU, donde se puede observar la tendencia de la participación de voluntarios entre los años 2011 y 2014.

En ambos gráficos se muestra la participación de voluntarios en los últimos cinco años. Los datos fueron procesados por el área de coordinación del voluntariado de acuerdo con los registros que constan desde el año 2011, cuando se comenzaron a efectuar los mismos. Vale aclarar que en el año 2011 se observó un número elevado de voluntarios que desciende de manera importante en el año 2012; esto se debe a que aún se contabilizaban estudiantes que integraban los Proyectos de Extensión de Cátedra (PEC), cuya característica era la participación del conjunto de estudiantes de la cátedra. Actualmente, ese espacio ha sido enriquecido y transformado en un área específica denominada Incorporación Curricular de la Extensión y se llevan a cabo las Prácticas de Extensión de Educación Experiencial.
Gráfico 1. Tendencia de voluntarios

\begin{tabular}{lllll}
\hline$\%$ & 2011 & 2012 & 2013 & 2014 \\
\hline
\end{tabular}

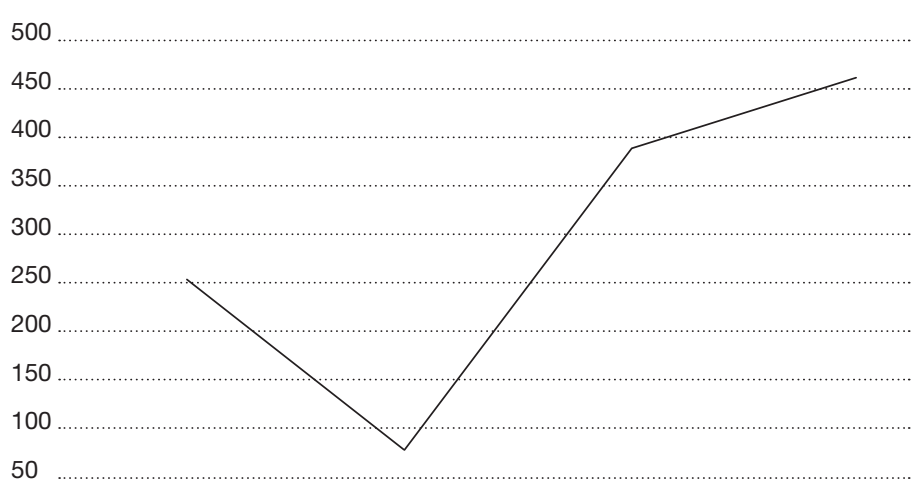

0

Fuente: SIPPE, de la Secretaría de Extensión, UNL.

Gráfico 2

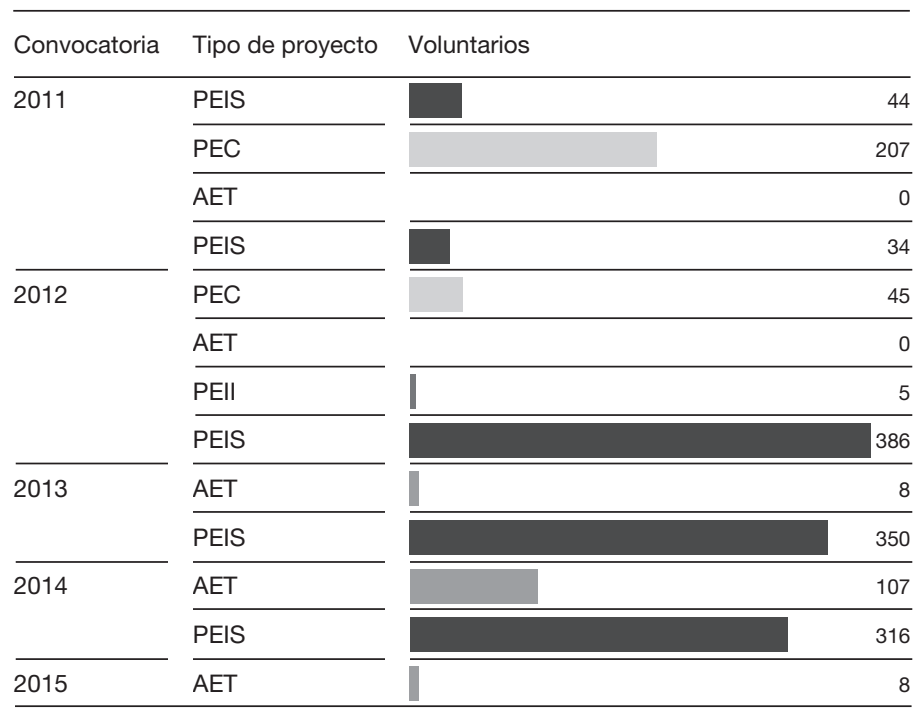

Referencias de siglas

$\square$ PEIS (Proyectos de Interés Social)

AET (Acciones de Extensión al Territorio)

PEII (Proyectos de Extensión de Interés Institucional)

$\square$ PEC (Proyectos de Extensión de Cátedra).

Fuente: SIPPE, de la Secretaría de Extensión, UNL. 


\section{A modo de cierre}

En estos últimos años hemos asistido a un crecimiento notable en el esfuerzo realizado para que estas experiencias de voluntariado formen parte de la cultura de la comunidad universitaria.

Si bien mucho se ha avanzado en los grados de institucionalización del voluntariado entendido como prácticas solidarias y educativas, observamos que aún resta desarrollar y fortalecer mecanismos de gestión y evaluación que nos permitan sistematizar y evaluar lo que acontece en el marco de estos procesos. Reconocemos la necesidad de contar con un instrumento que nos permita caracterizar, reflexionar y analizar el modelo de intervención con relación a procesos, técnicas, aprendizajes, intercambios que las mismas generan, así como en cuanto a los agentes sociales que participan de manera activa.

Si la Reforma Universitaria de 1918 apuntó, entre otros, a producir cambios en los modos de enseñanza y a alcanzar una universidad menos elitista y más inclusiva, hoy es necesario que volvamos a releer en el contexto actual aquellos históricos principios que iluminaron la educación superior universitaria. Es una deuda pendiente iniciar un proceso de análisis y reflexión teórica sobre la posición epistemológica que subyace en la concepción sobre la enseñanza y el aprendizaje. Como reformistas, adherimos a una práctica docente entendida como práctica social, práctica política, práctica humanística que se condense en una praxis educativa, que guarde en ella misma un potencial transformador, que confronte el paradigma tradicional de universidad y rompa con las lógicas positivistas impregnadas en muchos docentes.

El desafío está planteado. Lograr una mayor integración de la docencia, la investigación y la extensión sin dudas requiere del desarrollo de concepciones ideologías que rompan con concepciones cientificistas y de la profundización de políticas que fortalezcan esta integración de las funciones sustantivas. El Programa de Voluntariado universitario como programa de extensión es uno de los caminos posibles.

\section{Referencias bibliográficas}

Álvarez Méndez, J. M. (2003). La evaluación a examen. Buenos Aires: Miño y Dávila. Camilloni, A. (2011). La inclusión de la extensión en la formación de los estudiantes de la Universidad Nacional del Litoral. Revista +E, (1). Santa Fe: Ediciones UNL. Morin, E. (2000). Los siete saberes necesarios para la educación del futuro. Buenos Aires: Nueva Visión.

Rafaghelli, M. (2013). La dimensión pedagógica de la extensión. En Menéndez, G. y otros, Integración docencia y extensión. Otra forma de enseñar y aprender. Santa Fe: Ediciones UNL. 